\title{
INVENTARIO CAMPESINO DE MAMÍFEROS MEDIANOS Y GRANDES PARA UN PAISAJE DE LA SELVA LACANDONA
}

Rodolfo Palacios-Silva ${ }^{1}$, Eduardo Espinoza-Medinilla ${ }^{2}$, Juan García-Chávez ${ }^{3}$ y Sergio LóPez-MendozA ${ }^{4}$

${ }^{1}$ Escuela de Ingeniería Ecológica. Facultad de Ingeniería. Universidad de Ciencias y Artes de Chiapas. Km 1.5 terracería Nueva Palestina-Sa'kts'i. Nueva Palestina, Ocosingo, 29954 Chiapas, México.

2Posgrado en Desarrollo Sustentable. Facultad de Ingeniería. Universidad de Ciencias y Artes de Chiapas. Ciudad Universitaria Libramiento Norte-Poniente 1150 C. P. 29047, Tuxtla Gutiérrez, Chiapas, México.

${ }^{3}$ Escuela de Biología. Benemérita Universidad Autónoma de Puebla. Edificio 112A, Ciudad Universitaria, Av. San Claudio s/n, Col. San Manuel, Puebla, 72570 Puebla, México.

${ }^{4}$ Escuela de Biología. Facultad de Ciencias Biológicas. Universidad de Ciencias y Artes de Chiapas. Ciudad Universitaria Libramiento Norte-Poniente 1150 C. P. 29047, Tuxtla Gutiérrez, Chiapas, México.

Autor de correspondencia: Rodolfo Palacios-Silva; rodolfo.palacios@unicach.mx

\section{RESUMEN}

Se obtuvo un inventario potencial de los mamíferos medianos y grandes de la Sierra La Cojolita, un fragmento importante de vegetación natural remanente de la Selva Lacandona no protegido. El inventario fue realizado a partir de entrevistas con campesinos tseltales. El listado está conformado por 34 especies, 15 de ellas bajo algún tipo de protección de acuerdo a la NOM-059-SEMARNAT-2010. A pesar del reconocimiento de un número considerable de especies, la principal valoración de los mamíferos fue como fuente de alimento así como por sus afectaciones a la milpa o los animales de corral y de granja. De acuerdo a las entrevistas, la Sierra La Cojolita, un área no protegida, es un potencial reservorio de muchas especies de mamíferos y la cacería, aunada a la tala clandestina y la deforestación indiscriminada, sus principales amenazas.

Palabras clave: cacería, Chiapas, listado faunístico, valoración de la fauna, Sierra La Cojolita. 


\section{ABSTRACT}

A preliminary inventory of medium and large mammals for Sierra La Cojolita, an important unprotected remnant of tropical rainforest in the Lacandona region, was obtained. The inventory was created from interviews with peasants of the tseltal ethnic group. The list includes 34 species, 15 of them listed under some category of protection within the NOM-059-SEMARNAT-2010. Despite the recognition of many species, the main perception of the local mammals was as food source and their impact on agriculture and poultry. According to the interviews, Sierra La Cojolita is a reservoir of many mammal species, and hunting, illegal logging and indiscriminate deforestation its main threats.

Keywords: Chiapas, faunistic list, hunting, appreciation of fauna, Sierra La Cojolita.

\section{INTRODUCCIÓN}

El uso del territorio produce un conjunto de interacciones cotidianas entre las comunidades humanas y la fauna (Racero-Casarrubia, 2008; Parra-Colorado et al., 2014). El reconocimiento y la valoración de los mamíferos por parte de los pobladores de un territorio en específico puede ser el punto de partida para identificar recursos de interés social (Aldana et al., 2006; Racero-Casarrubia, 2008) y podría aportar información base para desarrollar estrategias de conservación a nivel local (Pérez-Irineo y Santos-Moreno, 2012).

La Selva Lacandona es uno de los remanentes más importantes de selva alta perennifolia en México y uno de los sitios con mayor riqueza de mamíferos en Mesoamérica (Medellín y Equihua, 1998; Cruz-Lara et al., 2004; Escobedo et al., 2005). En la actualidad, muchas de las especies de mamíferos que habitan la Selva Lacandona se encuentran es riesgo por la pérdida y fragmentación de su hábitat, así como por la presión ejercida por la cacería furtiva y el tráfico de fauna (Medellín, 1994; Tejeda, 2002). La persistencia de las poblaciones locales dependerá de acuerdos locales de conservación que deberán sumarse a los ya existentes.

En la porción mexicana de la Selva Lacandona se han establecido siete áreas naturales protegidas como política de conservación biológica: tres Áreas de Protección de Flora y Fauna (Nahá, Metzabok y Chan-kín); dos Monumentos Naturales (Bonampak y Yaxchilán) y dos Reservas de la Biosfera (Lacantún y Montes Azules), que en conjunto suman 419,450 hectáreas. Considerar la conectividad del paisaje permitiría que las áreas naturales protegidas de la Selva Lacandona se manejen como modelos fuente-sumidero (Naranjo y Bodmer, 2007).

La Selva Lacandona es habitada por poblaciones rurales que practican principalmente la agricultura y la ganadería de subsistencia, y para quienes los fragmentos de vegetación natural remanente son fuente de caza y recolección. Particularmente, los bienes comunales de la zona Lacandona fueron formados por tres etnias indígenas (Lacandón, Ch'ol y Tesltal) distribuidas en menos de una decena de poblaciones; su territorio incluye a las áreas naturales de Yaxchilán, Chan-kin, Bonampak, una buena parte de Montes Azules, así como dos regiones no protegidas conocidas como las Cañadas y Sierra La Cojolita (Tejeda-Cruz, 2002).

En este trabajo se proporciona información sobre el reconocimiento de especies de mamíferos medianos y grandes de un territorio que contiene un importante remanente de selva alta perennifolia con bajo grado de perturbación; además, recaba aspectos básicos de la valoración de los habitantes locales hacia la mastofauna de la región. Esta información es útil para la orientación de acciones de preservación para áreas sin algún decreto federal o estatal de protección en la región de la Selva Lacandona y la conservación local de los mamíferos presentes. 


\section{MÉTODO}

\section{ZONA DE ESTUDIO}

El clima predominante en la región lacandona es cálido y húmedo, con una precipitación y temperatura anual de aproximadamente $2,200 \mathrm{~mm}$ y $22^{\circ} \mathrm{C}$, respectivamente. El paisaje de referencia es conocido localmente como Sierra La Cojolita $\left(91^{\circ} 04^{\prime} 53^{\prime \prime} \mathrm{O}, 16^{\circ} 52^{\prime} 06^{\prime \prime} \mathrm{N}\right)$ dentro de la cuenca Usumacinta adyacente a las Áreas Naturales Protegidas Montes Azules, Yaxchilán y Bonampak (Figura 1). El territorio pertenece a los Bienes Comunales de la Zona Lacandona, constituido por varias comunidades indígenas (Tejeda, 2002). Más del 65\% de Sierra La Cojolita presenta vegetación natural remanente, principalmente selva alta perennifolia y menos del $35 \%$ restante equivale a acahuales, potreros y campos de cultivo (maíz y frijol, principalmente), así como servicios (caminos, carreteras, líneas de energía eléctrica). En esta área se presentan altitudes desde los 100 a los $900 \mathrm{msnm} ; 78 \%$ de su superficie es moderadamente plana $(<15 \%)$ mientras que el $20 \%$ y el $2 \%$ presentan una pendiente moderada y pronunciada, respectivamente.

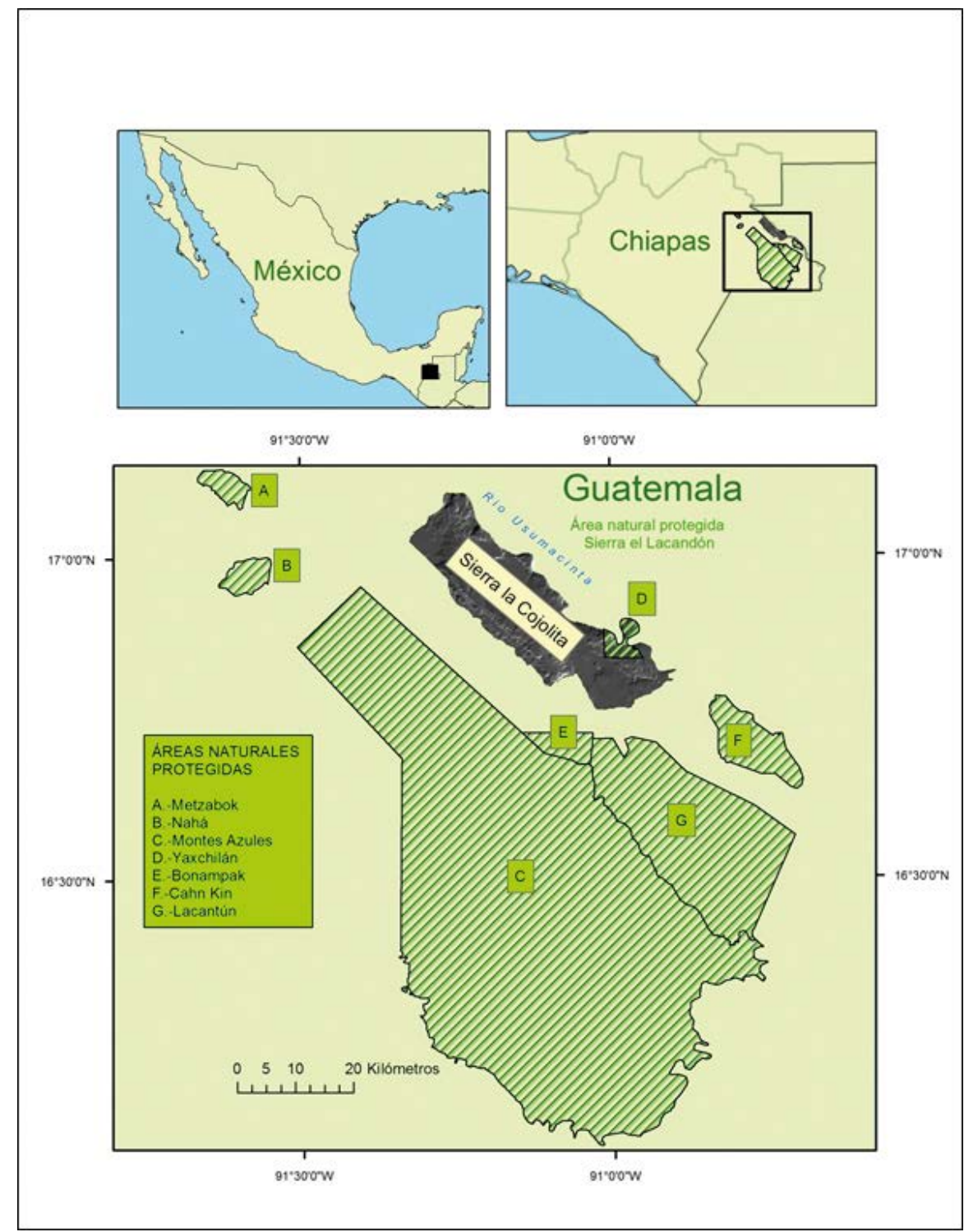

Figura 1. Ubicación de la Sierra la Cojolita. 


\section{ENTREVISTAS}

El estudio se realizó con información proporcionada por habitantes de la comunidad de Nueva Palestina, una comunidad indígena de aproximadamente 12,000 habitantes distribuidos en un poco más de 1,350 viviendas, regidos por sus usos y costumbres y con una economía basada en la producción primaria de subsistencia (CONAPO, 2001). Como criterio de inclusión para la realización de las entrevistas se identificó a nivel familiar a pobladores de la comunidad de Nueva Palestina que dijeran poseer terreno en la Sierra La Cojolita o zonas aledañas. Posteriormente al reconocimiento de propiedad del territorio se seleccionaron 75 unidades familiares y durante la visita a los hogares, se realizó una entrevista a algún miembro masculino de al menos 18 años de edad. Las entrevistas se desarrollaron en tseltal (con ayuda de traductores) y español, entre los meses de septiembre y diciembre del 2015.

A cada entrevistado se le mostraron ocho láminas con imágenes a color de mamíferos medianos y grandes, tomadas de la obra de Aranda (2012). Las láminas fueron agrupadas en marsupiales (6 figuras); monos y hormigueros (5 figuras); cánidos y mustélidos ( 7 figuras); felinos (6 figuras); zorrillos y armadillos (11 figuras); mapaches y otros (6 figuras); herbívoros pequeños y grandes (9 figuras) y liebres y conejos (4 figuras). Cada lámina incluyó al menos una especie no registrada en la zona de estudio (e.g. Linx rufus, Odocoileus hemionus, Taxidea taxus, Alouatta palliata, para las láminas de los felinos, herbívoros, mustélidos y monos, respectivamente) a manera de indicador de veracidad para las respuestas (llamado en el texto como testigo). Es importante mencionar que debido a que todos los marsupiales mexicanos referidos en la obra de Aranda tienen presencia en la región, esta agrupación no presentó imágenes testigo. Para cada especie se solicitó al entrevistado que indicara si lo reconocía (si había tenido alguna experiencia cercana, caza, mascota, etc.) y si sabía de alguna utilidad (alimento, mascota, ornamental, artesanal, medicinal o comercio).

\section{ANÁLISIS}

El inventario se obtuvo con el listado de especies que fueron reconocidas por los entrevistados. En este trabajo se utilizó como estimador de la eficiencia de muestreo la curva de acumulación de especies obtenida con las entrevistas a partir de un remuestreo completo, sin repetición, para 3,000 simulaciones. Aunque los métodos de remuestreo son criticados como técnica de extrapolación, son también comúnmente utilizados para estimar la diversidad alfa (Colwellet al., 2004). El análisis se realizó utilizando la librería rich del paquete de cómputo estadístico r-cran ${ }^{\mathrm{TM}}$ (Rossi, 2011).

\section{RESULTADOS}

De las 46 especies locales referidas en las láminas, los entrevistados reconocieron 34 (Cuadro 1). El valor de la mediana de especies mencionadas por entrevistado fue de seis, el mínimo de tres y el máximo de nueve. Siete especies fueron reconocidas en una sola ocasión: armadillo cola desnuda, mico dorado, puma, leoncillo, jaguar, comadreja y martucha. Un poco más del $40 \%$ de las especies mencionadas se encuentran bajo algún tipo protección según la NOM-059-SEMARNAT-2010; 10 están en peligro de extinción, tres amenazadas y dos bajo protección especial (SEMARNAT, 2010). La curva de acumulación de especies muestra que el incremento de especies no mencionadas es pequeño a partir de la entrevista 30 (Figura 2). La curva de rarefacción para los métodos de remuestreo bootstrap es mayor por dos especies respecto a la riqueza observada. En cuanto a su utilidad, un poco más del $55 \%$ de las especies mencionadas fueron señaladas como fuente de alimento; como mascota únicamente se mencionó a los conejos; como especies ornamentales se señaló a los venados y felinos. Las especies utilizadas con fines artesanales fueron venados, pecaríes y felinos; mientras que las únicas especies mencionadas como medicinales fueron las dos especies de mono (Cuadro 2). 
Cuadro 1. Listado campesino de las especies de mamíferos medianos y grandes en la Sierra de la Cojolita.

\begin{tabular}{|c|c|c|c|c|}
\hline Familia & Nombre científico & Nombre común & Frecuencia & NOM 059 ECOL \\
\hline \multirow[t]{4}{*}{ Didelphidae } & Caluromys derbianus & Tlacuachillo dorado & 3 & $\operatorname{Pr}$ \\
\hline & Didelphis marsupialis & Tlacuache común & 20 & \\
\hline & D. virginiana & Tlacuache común & 8 & \\
\hline & Philander opossum & Tlacuache cuatro ojos & 6 & \\
\hline \multirow[t]{2}{*}{ Dasypodidae } & Dasypus novemcinctus & Armadillo & 40 & \\
\hline & Cabassous centralis & Armadillo cola desnuda & 1 & $P$ \\
\hline Cyclopedidae & Cyclopes didactylus & Mico dorado & 1 & $\mathrm{P}$ \\
\hline Myrmecophagidae & Tamandua mexicana & Oso hormiguero & 5 & $\mathrm{P}$ \\
\hline \multirow[t]{2}{*}{ Atelidae } & Alouatta pigra & Mono aullador & 33 & $\mathrm{P}$ \\
\hline & Ateles geoffroyi & Mono araña & 31 & $\mathrm{P}$ \\
\hline \multirow[t]{2}{*}{ Leporidae } & Sylvilagus brasilensis & Conejo & 18 & \\
\hline & S. floridanus & Conejo castellano & 37 & \\
\hline \multirow[t]{5}{*}{ Felidae } & Leopardus pardalis & Ocelote & 6 & $\mathrm{P}$ \\
\hline & Leopardus wiedii & Tigrillo & 23 & $\mathrm{P}$ \\
\hline & Puma concolor & Puma & 1 & $\mathrm{P}$ \\
\hline & Herpailurus yagoarundi & Leoncillo & 1 & A \\
\hline & Pantera onca & Jaguar & 1 & $\mathrm{P}$ \\
\hline Canidae & Urocyon cinereoargenteus & Zorra gris & 17 & \\
\hline \multirow[t]{3}{*}{ Mustelidae } & Lontra longicaudis & Nutria & 14 & A \\
\hline & Eira barbara & Viejo de monte & 13 & $\mathrm{P}$ \\
\hline & Mustela frenata & Comadreja & 1 & \\
\hline \multirow[t]{2}{*}{ Mephitide } & Conepatus semistriatus & Zorrillo espalda blanca & 17 & \\
\hline & Spilogale angustifrons & Zorrillo manchado & 2 & \\
\hline \multirow[t]{3}{*}{ Procyonidae } & Nasua narica & Coatí & 5 & \\
\hline & Procyon lotor & Mapache & 3 & \\
\hline & Potos flavus & Martucha & 1 & $\operatorname{Pr}$ \\
\hline Tapiridae & Tapirus bairdii & Tapir & 7 & $\mathrm{P}$ \\
\hline \multirow[t]{2}{*}{ Tayassuidae } & Pecari tajacu & Pecarí de collar & 21 & \\
\hline & Tayassu pecari & Pecarí de labios blancos & 2 & \\
\hline \multirow[t]{2}{*}{ Cervidae } & Mazama temama & Temazate rojo & 16 & \\
\hline & Odocoileus virginianus & Venado cola blanca & 22 & \\
\hline Sciuridae & Sciurus deppei & Ardilla & 35 & \\
\hline Dasyproctyidae & Dasyprocta punctata & Guaqueue & 2 & \\
\hline Cuniculiade & Cuniculus paca & Tepezcuintle & 52 & \\
\hline
\end{tabular}

**Las categorías de riesgo de extinción según la NOM-059-ECOL son: P, peligro a la extinción; A, amenazadas; Pr, protección especial. 


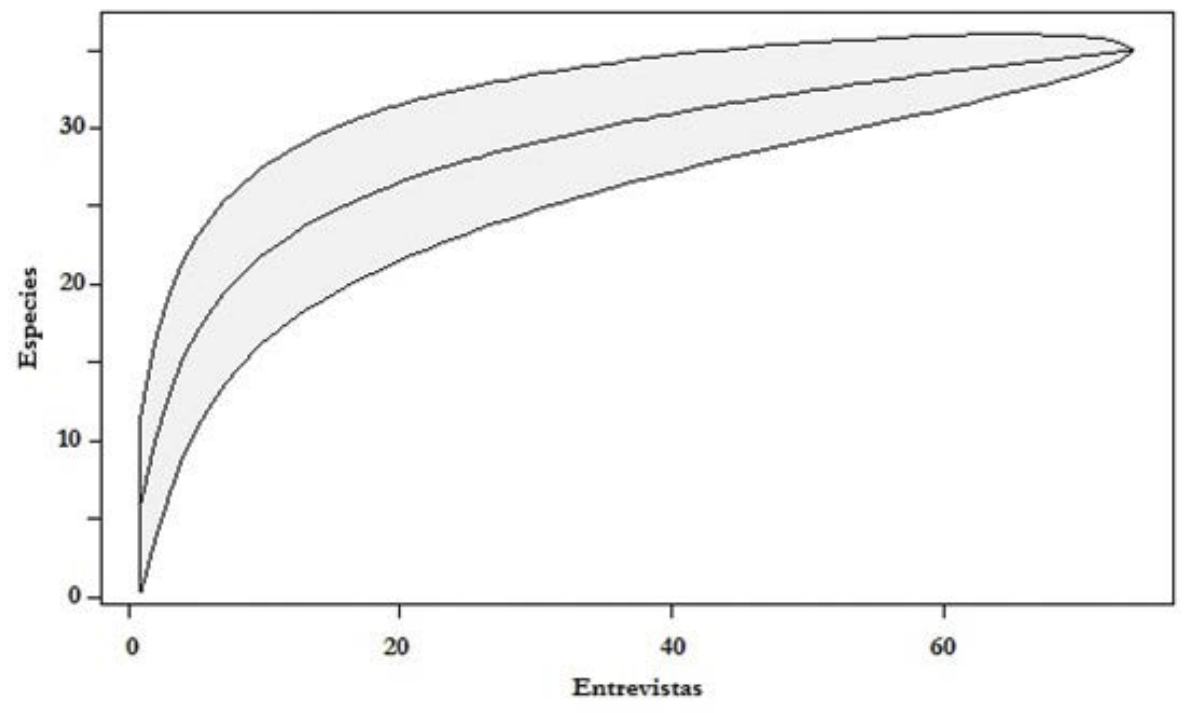

Figura 2. Curva de acumulación de especies con relación al numero de entrevistas con intervalos confianza al $95 \%$.

Cuadro 2. Usos referidos de los mamíferos.

\begin{tabular}{|c|c|c|c|c|c|c|}
\hline \multirow[t]{2}{*}{ Nombre científico } & \multicolumn{6}{|c|}{ Usos } \\
\hline & Alimento & Mascota & Ornamental & Artesanal & Medicinal & Comercio \\
\hline Didelphis marsupialis & $x$ & & & & & \\
\hline D. virginiana & $X$ & & & & & \\
\hline Philander opossum & $x$ & & & & & \\
\hline Dasypus novemcinctus & $x$ & & & & & \\
\hline Cabassous centralis & $x$ & & & & & \\
\hline Alouatta pigra & $x$ & & & & $X$ & \\
\hline Ateles geoffroyi & $x$ & & & & $x$ & \\
\hline Sylvilagus floridanus & $X$ & $x$ & & & & \\
\hline Leopardus pardalis & $X$ & & $x$ & $\mathrm{X}$ & & $x$ \\
\hline L. wiedii & $x$ & & $\mathrm{X}$ & $X$ & & $x$ \\
\hline Puma concolor & & & $x$ & $x$ & & \\
\hline P. yagoarundi & & & $\mathrm{X}$ & $\mathrm{X}$ & & \\
\hline Pantera onca & & & $\mathrm{X}$ & $\mathrm{X}$ & & \\
\hline Nasua narica & $X$ & & & & & \\
\hline Procyon lotor & $x$ & & & & & \\
\hline Tapirus bairdii & $x$ & & & & & \\
\hline Pecari tajacu & $x$ & & & $x$ & & \\
\hline Tayassu pecari & $x$ & & & $X$ & & \\
\hline Mazama temama & $x$ & & $\mathrm{X}$ & $\mathrm{X}$ & & \\
\hline Odocoileus virginianus & $X$ & & $x$ & $X$ & & \\
\hline Dasyprocta mexicana & $x$ & & & & & \\
\hline Cuniculus paca & $X$ & & & & & \\
\hline
\end{tabular}




\section{DISCUSIÓN Y CONCLUSIONES}

En este estudio se recopiló un inventario de 34 especies de mamíferos de la Sierra La Cojolita elaborado a partir del conocimiento local. Este trabajo muestra que los entrevistados reconocen al conjunto de especies cercano al potencialmente esperado. Considerando el listado elaborado para Yaxchilán (Escobedo et al., 2005), un área adyacente a la zona de estudio, no fueron reconocidos: el tlacuachín (Marmosa mexicana), el grisón (Galictis vittata), el cacomixtle tropical (Bassariscus sumichrasti) y el puercoespín tropical (Sphiggurus mexicanus), especies que tienen una alta probabilidad de distribución en la zona de estudio. Esto se puede deber a que son animales crípticos. Además de contar con traductores, las láminas con dibujos fueron un recurso de utilidad para la identificación de mamíferos. Un indicador en este trabajo sobre la disponibilidad de los entrevistados a proporcionar una respuesta veraz, fue la ausencia total de mención de especies que no tienen registro de distribución en la zona de estudio.

Dado que la valoración de las especies puede estar en función de los usos que se les dé, podría entenderse que la cacería de susbsistencia genera una apreciación favorable de las especies que proveen alimento. Así mismo, al considerar a la cacería como una actividad recreativa, la valoración de los entrevistados hacia los mamíferos es positiva. A pesar del amplio reconocimiento de las especies esperadas en la Sierra La Cojolita de manera individual, los entrevistados manifestaron un uso muy reducido de las especies, ya que los asocian principalmente a fuentes de alimento y amenazas para la cosecha y animales de corral; a diferencia de otros estudios similares (González-Bocanegra et al., 2011; Naranjo et al., 2014), donde los mamíferos tienen usos medicinales, para mascotas y comercio o de ornato. Por ello el tepezcuintle, armadillo, pecarí de collar y venado cola blanca, así como el mapache fueron cuatro de las especies más mencionadas por los entrevistados, así como los felinos en general.

La identificación de un número considerable de especies en la región por parte de la población tseltal puede ser evidencia de que el uso de la fauna silvestre en esta porción de selva es manifestación de condiciones de pobreza (Lorenzo et al., 2007). La precariedad de los sistemas agropecuarios en la Selva Lacandona no genera condiciones óptimas de producción alimenticia (Tejeda-Cruz, 2009), por lo que la presión ejercida sobre las poblaciones de mamíferos locales es natural. La pérdida de conocimiento tradicional de los pueblos indígenas que habitan la Selva Lacandona, por la reubicación geográfica de los asentamientos y la veloz incorporación a la economía de mercado, ha provocado que la importancia de los mamíferos como generadores de servicios ecosistémicos pase desapercibida. Debido a que el uso del territorio refleja una acción socioambiental, originado de un conjunto de patrones de conducta entre el conocimiento y la actitud ambiental (Akca et al., 2007), es importante reconfigurar la apropiación del territorio para conducir a un uso racional de la fauna local. La Educación Ambiental dirigida al reconocimiento del paisaje debe ser una herramienta que desarrolle el sentido de empoderamiento responsable en la comunidad (Sauvé, 2002).

La Selva Lacandona es una región prioritaria para la conservación biológica en Mesoamérica (Myers et al., 2000) y particularmente importante para la conservación de los mamíferos mexicanos (Vázquez et al., 2009). El agresivo cambio de uso de suelo en la región por la apertura de pastizales y campos de cultivo ha tenido efectos negativos en la diversidad en general por la fragmentación y la pérdida de calidad del hábitat (Medellín, 1994; Medellín y Equihua, 1998; Garmendia et al., 2013). La Sierra La Cojolita es un macizo de vegetación natural importante, sin embargo, la expansión de la frontera productiva ha comenzado a amenazar la integridad del sistema natural remanente. Sierra La Cojolita tiene una ubicación geográfica trascendental ya que conecta varias áreas naturales protegidas en la porción mexicana de la Selva Lacandona. De continuar con la deforestación indiscriminada, la tala ilegal y la cacería sin regulación, el paisaje de la Cojolita podría perder localmente varias de las especies de mamíferos presentes. 
Generalmente las entrevistas son un método que provoca desconfianza respecto a otros métodos indirectos y directos para la detección de especies (Zapata-Ríos et al., 2006). Sin embargo, este trabajo puede ser el punto de partida para explorar la valoración hacia los mamíferos como recurso de interés social en la población de estudio (Aldana et al., 2006; Racero-Casarrubia, 2008). Los campesinos tseltales, presentan una cercanía considerable a la reserva comunal respecto a los demás grupos instalados en las inmediaciones de la Sierra La Cojolita y además, son el grupo más numeroso con una proporción poblacional alrededor de los doce mil habitantes (INEGI, 2010). La capacidad de carga de la reserva se encuentra actualmente comprometida por la presión humana, es por ello que resulta necesario diseñar estrategias para la regulación de la extracción de los recursos de la Sierra La Cojolita.

En conclusión, para el funcionamiento de la Sierra La Cojolita como reserva comunal es indispensable promover y aplicar estrategias de conservación desde la convergencia de instituciones educativas, instituciones gubernamentales y no gubernamentales, en conjunto con la población local. Las prácticas co-participativas son una estrategia efectiva para el manejo de los recursos naturales (Bocco et al., 2000). Uno de los primeros pasos es la generación de información sobre la riqueza de especies y el uso del recurso en un contexto de una unidad espacial definida. El presente trabajo podría ser considerado como un paso inicial para la conformación formal de la zona como área natural protegida reconocida por los habitantes de la región, quienes son los que verdaderamente importan al momento de realizar prácticas de manejo y conservación en cualquier lugar. El involucramiento es una exigencia interna de la naturaleza del ser humano (Kliksberg, 1999). Este primer intento por catalogar las especies en la región presenta información asociada a los usos principales para proponer programas de manejo de las especies identificadas. A través de la valoración de la fauna, se pueden identificar aquellas que necesiten reorientación a través de programas de Educación Ambiental dirigidos a especies catalogadas como peligrosas o desagradables, así como trabajar en la construcción de propuestas para minimizar el conflicto hombre-fauna.

\section{AGRADECIMIENTOS}

A los estudiantes de la escuela de Ingeniería Ecológica de la UNICACH que participaron en la traducción de las entrevistas, a las personas que facilitaron la información y a Patricia Figueroa Esponda por la revisión de este manuscrito.

\section{LITERATURA CITADA}

Akca H., M. Sayili y M. Yilmazcoban. 2007. Rural Awareness of Environmental Issues: the Case of Turkey. Polish Journal of Environmental Studies, 16:177-182.

Aldana, N., M. Díaz-Porres, A. Feijoo y C. Zuñiga. 2006. Valoración del uso de la fauna silvestre en el municipio de Alcalá, Valle del Cauca. Universidad Tecnológica de Pereira. Scientia et Technica, 12:291-296.

Aranda, M. 2012. Manual para el rastreo de mamíferos silvestres de México. conABıo, México, Distrito Federal.

Bocco, G., A. Velázquez y A. Torres. 2000. Ciencia, comunidades indígenas y manejo de recursos naturales. Un caso de investigación participativa en México. Interciencia, (25):64-70.

Castaño, J, y J. Corrales. 2011. Mamíferos de la cuenca del río La Miel (Caldas): diversidad y uso cultural. Boletín Científico del Museo de Historia Natural, 14:56-75.

Colwell R., Ch. Xuan Mao y J. Chang. 2004. Interpolating, extrapolating, and comparing incidence-based species accumulation curves. Ecology, 85:2717-2727. 
CONAPO. 2001. Índice de marginación 2000. Consejo Nacional de Población. Cuernavaca, México.

Cruz-Lara, L., C. Lorenzo, L. Soto, E. Naranjo y N. Ramírez-Marcial. 2004. Diversidad de mamíferos en cafetales y selva mediana de las cañadas de la Selva Lacandona, Chiapas, México. Acta Zoológica Mexicana, 20:63-81.

Dirzo R., H. Young, M. Galetti, G. Ceballo, N. Isaac y B. Collen. 2014. Defaunation in the Anthropocene. Science, 345:401-405

Escobedo L., L. León, J. Arroyo-Cabrales y O. Polaco. 2005. Diversidad y abundancia de los mamíferos de Yaxchilán, municipio de Ocosingo, Chiapas. Pp. 283-298, in: Contribuciones mastozoológicas en homenaje a Bernardo Villa. (Sánchez-Cordero V. y R. Medellín, eds.) UNAM y CONABIO. México D. F.

Garmendia, A., V. Arroyo-Rodríguez, A. Estrada, E. Naranjo y K. Stoner. 2013. Landscape and patch attributes impacting medium- and large-sized terrestrial mammals in a fragmented rain forest. Journal of Tropical Ecology, 29:331-344.

Garcia-Alaniz, N., E. Naranjo y F. Mallory. 2010. Human-Felid Interactions in Three Mestizo Communities of the Selva Lacandona, Chiapas, Mexico: Benefits, Conflicts and Traditional Uses of Species. Human Ecology 38:451-457.

González-Bocanegra, K., E. Romero-Berny, M.C. Escobar-Ocampo e Y. García del Valle. 2011. Aprovechamiento de fauna silvestre por comunidades rurales en los humedales de Catazajá-La Libertad, Chiapas, México. Ra Ximhai, 7:219-230.

INEGI. 2010. Censo de Población y Vivienda. México: Instituto Nacional de Estádistica y Geografía. Disponible en: http://www.inegi.gob.mx

Kliksberg, B.1999. Seis Tesis no convencionales sobre participación. Revista de Estudios Sociales [en línea]: [Fecha de consulta: 10 de junio de 2017] Disponible en:<http://www.redalyc.org/ articulo.oa?id=81511266010> ISSN: 0123-885X

Lorenzo C., L. Cruz, E. Naranjo y F. Barragán. 2007. Uso y conservación de mamíferos silvestres en una comunidad de las cañadas de la Selva Lacandona, Chiapas, México. Etnobiología, 5:99-107.

Medellín, R. 1994. Mammals diversity and conservation in the Selva Lacandona, Chiapas, Mexico. Conservation Biology, 8:780-799.

Medellín, R. y M. Equihua. 1998. Mammal species richness and habitat use in rainforest and abandoned agricultural fields in Chiapas, Mexico. Journal of Applied Ecology, 35:13-23.

Myers, N., R. Mittermeier, C. Mittermeier, G. da Fonseca y J. Kent. 2000. Biodiversity hotspots for conservation priorities. Nature, 403:853-858.

Naranjo, E.J., M.M. Guerra, R.E. Bodmer y J.E. Bolaños.2004. Subsistence hunting by three ethnics groups of the Lacandon Forest, Mexico. Journal of Ethnobiology, 24:233.

Naranjo, E., J. Rangel y C. Tejeda-Cruz. 2014. El manejo comunitario de fauna silvestre como instrumento de conservación en Marqués de Comillas, Selva Lacandona, Chiapas. Pp. 149173, en: Gestión Territorial y Manejo de Recursos Naturales: Fauna silvestre y sistemas agropecuarios. (Medina, L., C. Tejeda-Cruz, A. Carrillo y T. Mila, eds.) Tuxtla Gutiérrez, Chiapas, México. Universidad Autónoma de Chiapas. 
Naranjo, E.J. y R.E. Bodmer.2007. Source-sink systems and conservation of hunted ungulates in the Lacandon Forest, Mexico. Biological Conservation, 138:412-420.

Parra-Colorado J., Á. Botero-Botero y C. Saavedra-Rodríguez. 2014. Percepción y uso de mamíferos silvestres por comunidades campesinas andinas de Génova, Quindío, Colombia. Boletín Científico del Museo Historia Natural, 18:78-93.

Pérez-Irineo G. y A. Santos-Moreno. 2012. Diversidad de mamíferos terrestres de talla grande y media de una selva subcaducifolia del noreste de Oaxaca, México. Revista Mexicana de Biodiversidad, 83:164-169.

Racero-Casarrubia, J., C. Vidal, O. Ruiz y J. Ballesteros. 2008. Percepción y patrones de uso de fauna silvestre por las comunidades indígenas Embera Katíos en la cuenca del rio san Jorge, zona amortiguadora del PNN-Paramillo. Revistas de Estudios Sociales, 31:118-131.

Rossi, J.P. 2011. Rich: An R package to analyse species richness. Diversity, 3:112-120.

Sauvé, L. 2002. Environmental education: possibilities and constraints. Connect, 27:1-4.

SEMARNAT. 2010. Norma Oficial Mexicana NOM-059-SEMARNAT-2010. Protección ambiental-Especies nativas de México de flora y fauna silvestres-Categorías de riesgo y especificaciones para su inclusión, exclusión o cambio-lista de especies en riesgo. Segunda edición. Diario oficial, 6 de marzo del 2002.

Tejeda, C. 2002. Apropiación social del territorio y política ambiental en la Selva Lacandona, Chiapas; el caso de Frontera Corozal, Comunidad Lacandona. Tesis de Maestría. Dirección de Centros Regionales. Universidad Autónoma Chapingo.

Tejeda-Cruz, C. 2009. Conservación de la biodiversidad y comunidades locales: conflictos en áreas naturales protegidas de la Selva Lacandona, Chiapas, México. Canadian Journal of Latin American and Caribbean Studies, 34:57-88.

Vázquez L., C. Bustamante-Rodríguez y D. Bahena. 2009. Area selection for conservation of Mexican mammals. Animal Biodiversity and Conservation, 32:29-39

Vignieri, S. 2014. Vanishing fauna. Science, 345:293-295.

Zapata-Ríos, G., G. Araguillin y J. Jorgenson. 2006. Caracterización de la comunidad de mamíferos no voladores en las estribaciones orientales de la cordillera del Kutukú, Amazonía ecuatoriana. Mastozoología Neotropical, 13:227-238. 